The Egyptian Journal of Hospital Medicine (April 2019) Vol. 75 (3), Page 2397-2405

\title{
Immunohistochemical Expression of Cyclooxygenase-2 (COX-2) in Breast Cancer ${ }^{1}$ Mahmoud Ismail Ahmed Nassar, ${ }^{2}$ Shaimaa M.M. Bebars, ${ }^{2}$ Rasha Mohamed Samir Said, ${ }^{2}$ Taghreed Mohammed Amin Mustafa
}

${ }^{1}$ Department of Pathology, Faculty of Medicine, Assiut University, ${ }^{2}$ Department of Pathology, Faculty of Medicine, Aswan University

*Corresponding author: Taghreed Mohammed Amin Mustafa, E-Mail: taghreed.amin2020@gmail.com, Mobile: +201094384015

\section{ABSTRACT}

Background: breast cancer (BC) is the most common type of diagnosed cancers in women. It is still the second leading cause of cancer-related death among women after lung cancer all over the world. Breast cancer is the first of top ten cancers in Egypt. It ranks as the first malignancy affecting females, contributing $30 \%$ of all female cancers. It affects 1 in 14 women during their life time.

Aim: This study investigated the association between cyclooxygenase-2 (COX-2) expression in female breast cancer versus the expression of ER, PR, as well as its association with other established prognostic indicators like age, tumor size, lymph nodal status, stage, grade, lymphovascular invasion, insitu component and histological subtype, and aims to validate the role of overexpression of COX-2 as a prognostic marker in female patients with breast cancer in Egypt.

Results: High significant correlation was found between lymph node metastasis, negative ER and PR cases and COX-2 expression. No significant correlation could be detected between age, tumor size, site, histologic type, grade, insitu component, LVI and COX-2 expression.

Conclusion: Cyclooxygenase-2 has poor prognostic parameter in breast cancer, as it is over expressed in majority of breast carcinoma, especially with lymph node metastasis, ER and PR negative hormone receptor.

Keywords: Breast cancer, Cyclooxygenase-2, prognostic marker, immunohistochemistry (IHC), ERIPR receptor, clincopathological data.

\section{INTRODUCTION}

Breast cancer is the most frequently diagnosed cancer and the most common cause of cancer death among women. It accounts for nearly (23\%) of all female cancers worldwide, with more than a million new cases each year. Although BC incidence rates in Egypt are substantially lower than the rates in the United States and other developed countries, breast cancer is the most common cancer among women in Egypt. Prognosis of breast cancer depends on different factors as tumor size, histologic type, stage, and molecular profile $^{(\mathbf{1})}$.

Cyclooxygenase (COX) group of enzymes are important for the conversion of arachidonic acid to prostaglandins. Cyclooxygenase-1 (COX-1) is constitutively expressed at a constant level throughout the cell cycle in most of the tissues. The inducible isoform, Cyclooxygenase-2 (COX-2), is often over expressed in breast cancer ${ }^{(2)}$.

Cyclooxygenase-2 derived metabolites may contribute to maintenance of premalignant hyperproliferation, transformation, tumor viability, growth, invasion and metastatic spread ${ }^{(3)}$. Cox-2 and its products, particularly prostaglandin E2 $\left(\mathrm{PGE}_{2}\right)$, act via classical cancer signaling pathways in primary tumor cells to promote tumorigenesis. Evidence has shed a spotlight not only on the tumor cell itself, but also the tumor microenvironment, especially macrophages in the tumor ${ }^{(4)}$. Over-expression of COX-2 has been detected in several malignancies including breast cancer, and contributes to carcinogenesis by stimulating cancer cell proliferation, inhibiting apoptosis, increasing invasiveness and modulating inflammation and im- munity ${ }^{(5)}$. Clinical studies have noted a reduced risk for breast, lung, prostate, and colon cancers after treatment with non-selectively COX-2 inhibition by non-steroidal anti-inflammatory drugs (NSAIDs) or selective COX-2 inhibition with COX-2 inhibitors ${ }^{(6)}$.

It has been reported that elevated COX-2 expression was more common in larger size breast cancer tumors with axillary lymph node metastasis ${ }^{(7)}$. More studies are needed to confirm the potential role of $\mathrm{COX} 2$ in breast cancer prognosis and studying the role of COX2 inhibitors in reducing the risk of breast cancer ${ }^{(8)}$.

\section{AIM OF THE WORK}

To study the expression of (COX-2) in breast cancer and the relationship of its expression with the clinicopathological data of the studied cases, including (age, size, site of tumor, histological type, grade, lymph node metastasis, stage of tumor, estrogen and progesterone positive or negative tumor).

\section{MATERIAL AND METHODS}

This retrospective study was carried out on 50 cases of Egyptian (BC) patients. These cases were selected from archives of Pathology department, Faculty of Medicine, Assuit University, Aswan University and private laboratories from January 2014 till reaching the target of cases. Cases were collected as paraffin embedded blocks. The selected cases included modified radical mastectomy (MRM) and conservative breast surgery either lumpectomy or wide excision with axillary evacuation. 
The study was approved by the Ethics Board of Aswan University and an informed written consent was taken from each participant in the study.

The following cases with breast cancer were not suitable for this study: true cut biopsy, breast excisions without axillary evacuation, lumpectomies without axillary evacuation, cases with no residual invasive carcinoma after pre-surgical (neoadjuvant) chemotherapy, cases consisting predominately of CIS with microinvasion, cases with massive necrosis or fibrosis and male patient.

Clinical data: Clinical data were collected from pathology reports including (age, site, size of tumor and type of surgery). Two serial sections from each tissue block were cut at a 4 microns thickness to be used as follows: One section was stained with routine Haematoxylin and Eosin $(\mathrm{H} \& \mathrm{E})$ to evaluate the histopathologic diagnosis and the other one was stained with COX2 antibodies.

Histopathological examination: All cases were examined using a light microscope. The following items were recorded: Histopathological type: according to WHO classification ${ }^{(9)}$. IDC (NST), ILC, mixed ductal and lobular carcinoma, mucinous carcinoma, medullary carcinoma. Histological grade: Nottingham histological score ${ }^{(10)}$. In situ component. Lymph node metastasis. Lympho vascular invasion. Staging of breast carcinoma (T.N.M classification for staging of breast cancer is provided before).

ER \& PR stained slides: All cases were examined for their ER \& PR status according to the American Society of Clinical Oncology/College of American Pathologists (ASCO/CAP) (11) guideline recommendations for IHC testing of ER\& PR in breast cancer.

Uninterpretable: if finding that no tumor nuclei are immune reactive, and that internal control or normal epithelial elements present in the sample lack any nuclear staining.

Immunohistochemical staining: Sections of 4 $\mu \mathrm{m}$ thickness, from each case were stained for Cyclooxygenase 2 (COX-2). Negative control slide was run by omitting the primary antibody. Positive control was colorectal carcinoma. The method used for immunostaining was streptavidin-biotin amplified system. The primary antibodies used is Rabbit monoclonal antibody against COX-2, cat. \#RM-9121-S0 (Thermo scientific, Fremont, CA 94538-6406, USA), $0.1 \mathrm{ml}$ concentrated with dilution of 1:100.

\section{Evaluation of COX-2 immunostaining:}

For the evaluation of cytoplasmic staining results for COX-2, a predefined scoring system based on the product of staining intensity and percentage of positive tumor cells was used ${ }^{(\mathbf{1 2})}$. Immunoreactivity for COX-2 in tumor cells was assessed using a scoring system based on staining intensity:

Staining 0 and $1+$ were classified as "COX-2 negative", whereas staining $2+$ and $3+$ as "COX-2 positive" (13).

Confidentiality of the data:
No encroach on private data of the patient, as blocks of tumor tissue are going to be signed with serial code numbers instead of the name of the patient. Also, the results of the research will be used only in scientific aims.

\section{Statistical analysis of the data}

Data were collected, tabulated and statistically analyzed using a personal computer with Statistical Package for Social Science (SPSS) version 20.0. (Armonk, NY: IBM Corp) ${ }^{(\mathbf{1 4})}$. Qualitative data were described using number and percent. The KolmogorovSmirnov test was used to verify the normality of distribution Quantitative data were described using range (minimum and maximum), mean, standard deviation and median. Significance of the obtained results was judged at the $5 \%$ level ${ }^{(15)}$.

\section{The used tests were:}

1 - Chi- square test $\left(\mathbf{X}^{2}\right.$ - test): was used to compare between qualitative data such as different tumor grade and stage.

2 - Fisher's Exact or Monte Carlo correction: Correction for chi-square when more than $20 \%$ of the cells have expected count less than 5

3 - Student t-test: Used in comparing one quantitative variable and one qualitative variable if they were normally distributed.

4 - F-test (ANOVA): For normally distributed quantitative variables, to compare between more than two groups

5 - Mann Whitney test:For abnormally distributed quantitative variables, to compare between two studied groups

6 - Kruskal Wallis test: For abnormally distributed quantitative variables, to compare between more than two studied groups.

\section{Probability (P- value) ${ }^{(16)}$ : difference considered as} follow:

Statistically significant $(\mathrm{S})$ when $(\mathrm{P}<0.05)$.

Highly significant $(\mathrm{HS})$ when $(\mathrm{P}<0.01)$. Not significant (NS) when $(\mathrm{P}>0.05)$.

\section{RESULTS}

- Assessment of IHC expression of COX-2 was done in malignant epithelial cells for staining status, pattern and intensity.

- All positive cases showed cytoplasmic pattern of expression. 43 cases out of $50(86.0 \%)$ showed positive expression of COX-2, while 7 cases (14.0\%) showed negative expression (table 2).

- No significant correlation could be detected between age, tumor size, site, histologic type, grade, in situ component, LVI and COX2 expression (table 3 ).

- High significant correlation was found between lymph node metastasis $(\mathrm{P}=0.009)$, negative $\mathrm{ER}$ and $\mathrm{PR}$ cases $(\mathrm{P} \leq 0.001)$ and $\mathrm{COX} 2$ expression (table 4) 
Table (1): Distribution of the studied group as regard age, Site of tumor, histologic type, histologic grade, tumor size, in situ component, T.N.M and Lymphovascular invasion

\begin{tabular}{|c|c|c|}
\hline & No. & $\%$ \\
\hline \multicolumn{3}{|l|}{ Age (years) } \\
\hline$<50$ & 17 & 34.0 \\
\hline$\geq 50$ & 33 & 66.0 \\
\hline Total & $\mathbf{5 0}$ & $\mathbf{1 0 0 . 0}$ \\
\hline Min. - Max. & \multicolumn{2}{|c|}{$25.0-77.0$} \\
\hline Mean \pm SD. & \multicolumn{2}{|c|}{$52.18 \pm 12.21$} \\
\hline Median & \multicolumn{2}{|c|}{54.0} \\
\hline \multicolumn{3}{|l|}{ Site of tumor } \\
\hline UOQ & 31 & 62.0 \\
\hline $\begin{array}{l}\text { UIQ } \\
\end{array}$ & 4 & 8.0 \\
\hline LOQ & 3 & 6.0 \\
\hline LIQ & 5 & 10.0 \\
\hline Retro-areolar & 7 & 14.0 \\
\hline \multicolumn{3}{|l|}{ Diagnosis } \\
\hline Invasive duct carcinoma (NST) & 35 & 70.0 \\
\hline Invasive lobular carcinoma & 9 & 18.0 \\
\hline Medullary carcinoma & 3 & 6.0 \\
\hline Mucinous carcinoma & 2 & 4.0 \\
\hline Metaplastic carcinoma & 1 & 2.0 \\
\hline \multicolumn{3}{|l|}{ Histologic grade } \\
\hline $\mathrm{I}$ & 1 & 2.0 \\
\hline II & 37 & 74.0 \\
\hline III & 12 & 24.0 \\
\hline \multicolumn{3}{|l|}{ Tumor size } \\
\hline$<2$ & 6 & 12.0 \\
\hline $2-5$ & 36 & 72.0 \\
\hline$>5$ & 8 & 16.0 \\
\hline Min. - Max. & \multicolumn{2}{|c|}{$1.0-8.0$} \\
\hline Mean \pm SD. & \multicolumn{2}{|c|}{$3.73 \pm 1.67$} \\
\hline Median & \multicolumn{2}{|c|}{3.50} \\
\hline \multicolumn{3}{|l|}{ In situ component } \\
\hline No & 17 & 34.0 \\
\hline Yes & 33 & 66.0 \\
\hline \multicolumn{3}{|l|}{ Pathologic stage } \\
\hline T1 & 8 & 16.0 \\
\hline $\mathrm{T} 2$ & 31 & 62.0 \\
\hline $\mathrm{T} 3$ & 8 & 16.0 \\
\hline $\mathrm{T} 4$ & 3 & 6.0 \\
\hline N0 & 14 & 28.0 \\
\hline N1 & 9 & 18.0 \\
\hline $\mathrm{N} 2$ & 12 & 24.0 \\
\hline N3 & 15 & 30.0 \\
\hline \multicolumn{3}{|l|}{ Lymphovascular invasion } \\
\hline No & 34 & 68.0 \\
\hline Yes & 16 & 32.0 \\
\hline
\end{tabular}


Table (2): Distribution of the studied cases according to ER expression, PR expression, Cox2 expression

\begin{tabular}{|l|c|c|}
\hline & No. & \% \\
\hline ER & & 60.0 \\
\hline Negative & 30 & 8.0 \\
\hline Mild positive & 4 & 12.0 \\
\hline Moderate positive & 6 & 20.0 \\
\hline Strongly positive & 10 & \\
\hline PR expression & 32 & 64.0 \\
\hline Negative & 6 & 12.0 \\
\hline Mild positive & 3 & 6.0 \\
\hline Moderate positive & 9 & 18.0 \\
\hline Strongly positive & & \\
\hline Cox2 & 7 & $\mathbf{8 6 . 0}$ \\
\hline Negative & $\mathbf{4 3}$ & \\
\hline Positive & & \\
\hline
\end{tabular}

Table (3): Relation between Cox 2 expression and age, Site of tumor, histologic type, histologic grade, in situ component and Lymphovascular invasion

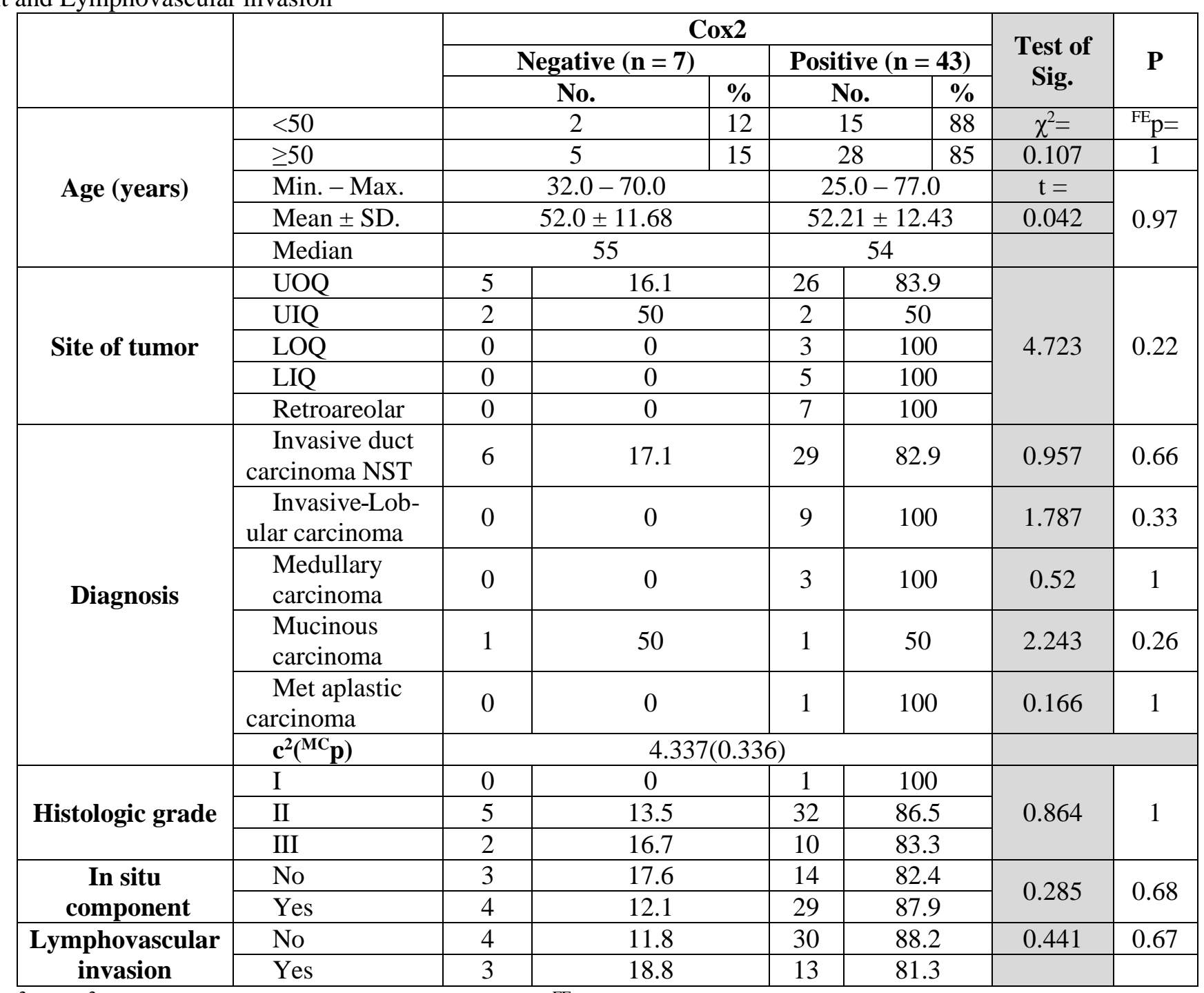

$\chi^{2}$, p: $\chi^{2}$ and $\mathrm{p}$ values for Chi square test $\quad{ }^{\mathrm{FE}} \mathrm{p}: \mathrm{p}$ value for Fisher Exact for Chi square test

${ }^{\mathrm{MC}} \mathrm{p}$ : $\mathrm{p}$ value for Monte Carlo for Chi square test $\mathrm{t}, \mathrm{p}$ : $\mathrm{t}$ and $\mathrm{p}$ values for Student t-test

- No significant statistical association could be detected between COX2 expression clinicopathological parame-

ter such as age, site of tumor, size, grade, insitu component and lymphovascular invasion. 
Table (4): Relation between Cox2 expression and lymph node status, Pathologic stage, ER expression and PR expression.

\begin{tabular}{|c|c|c|c|c|c|c|}
\hline & \multicolumn{4}{|c|}{$\operatorname{Cox} 2$} & \multirow{3}{*}{$\chi^{2}$} & \multirow{3}{*}{${ }^{\mathbf{F E}} \mathbf{p}$} \\
\hline & \multicolumn{2}{|c|}{$\begin{array}{c}\text { Negative } \\
(\mathbf{n}=7)\end{array}$} & \multicolumn{2}{|c|}{$\begin{array}{l}\text { Positive } \\
(n=43)\end{array}$} & & \\
\hline & No. & $\%$ & No. & $\%$ & & \\
\hline \multicolumn{7}{|c|}{ Lymph node status } \\
\hline Negative & 6 & 31.6 & 13 & 68.4 & \multirow{2}{*}{$7.962^{*}$} & \multirow{2}{*}{$0.009^{*}$} \\
\hline Positive & 1 & 3.2 & 30 & 96.8 & & \\
\hline \multicolumn{7}{|c|}{ Pathologic stage } \\
\hline \multicolumn{7}{|l|}{ Stage $T$} \\
\hline $\mathrm{T} 1$ & 0 & 0.0 & 8 & 100.0 & 1.719 & 0.693 \\
\hline $\mathrm{T} 2$ & 6 & 19.4 & 25 & 80.6 & & \\
\hline T3 & 1 & 12.5 & 7 & 87.5 & & \\
\hline $\mathrm{T} 4$ & 0 & 0.0 & 3 & 100.0 & & \\
\hline \multicolumn{7}{|l|}{ Stage N } \\
\hline N0 & 1 & 7.1 & 13 & 92.9 & $8.537^{*}$ & $0.010^{*}$ \\
\hline N1 & 1 & 11.1 & 8 & 88.9 & & \\
\hline $\mathrm{N} 2$ & 5 & 41.7 & 7 & 58.3 & & \\
\hline N3 & 0 & 0.0 & 15 & 100.0 & & \\
\hline \multicolumn{7}{|c|}{ ER expression } \\
\hline Negative & 0 & 0.0 & 30 & 100.0 & \multirow{4}{*}{$13.824^{*}$} & \multirow{4}{*}{$0.001^{*}$} \\
\hline Mild & 1 & 25.0 & 3 & 75.0 & & \\
\hline Moderate & 2 & 33.3 & 4 & 66.7 & & \\
\hline Strongly & 4 & 40.0 & 6 & 60.0 & & \\
\hline \multicolumn{7}{|c|}{ PR expression } \\
\hline Negative & 0 & 0.0 & 32 & 100.0 & \multirow{4}{*}{$21.165^{*}$} & \multirow{4}{*}{$<0.001^{*}$} \\
\hline Mild & 0 & 0.0 & 6 & 100.0 & & \\
\hline Moderate & 2 & 66.7 & 1 & 33.3 & & \\
\hline Strongly & 5 & 55.6 & 4 & 44.4 & & \\
\hline
\end{tabular}

$\chi^{2}, \mathrm{p}: \chi^{2}$ and $\mathrm{p}$ values for Chi square test $\quad{ }^{\mathrm{MC}} \mathrm{p}: \mathrm{p}$ value for Monte Carlo for Chi square test

*: Statistically significant at $\mathrm{p} \leq 0.05 \quad{ }_{\mathrm{FE}} \mathrm{p}$ : $\mathrm{p}$ value for Fisher Exact for Chi square test

High significant correlation was found between lymph node metastasis, negative ER and PR cases and COX2 expression.

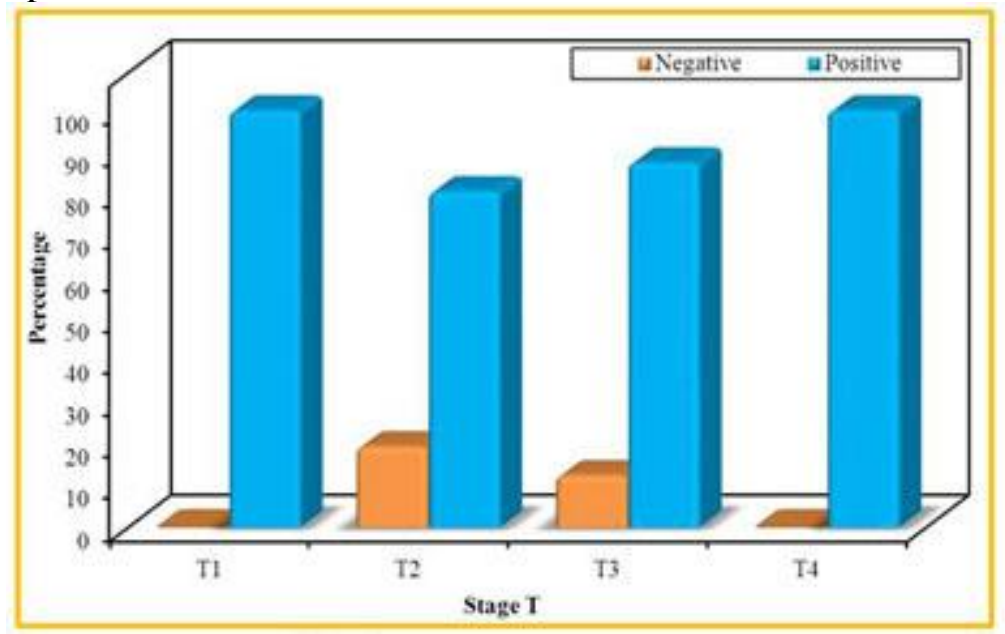

Figure (1): Relation between COX2 expression and stage T. 
Immunohistochemical Expression of Cyclooxygenase-2...

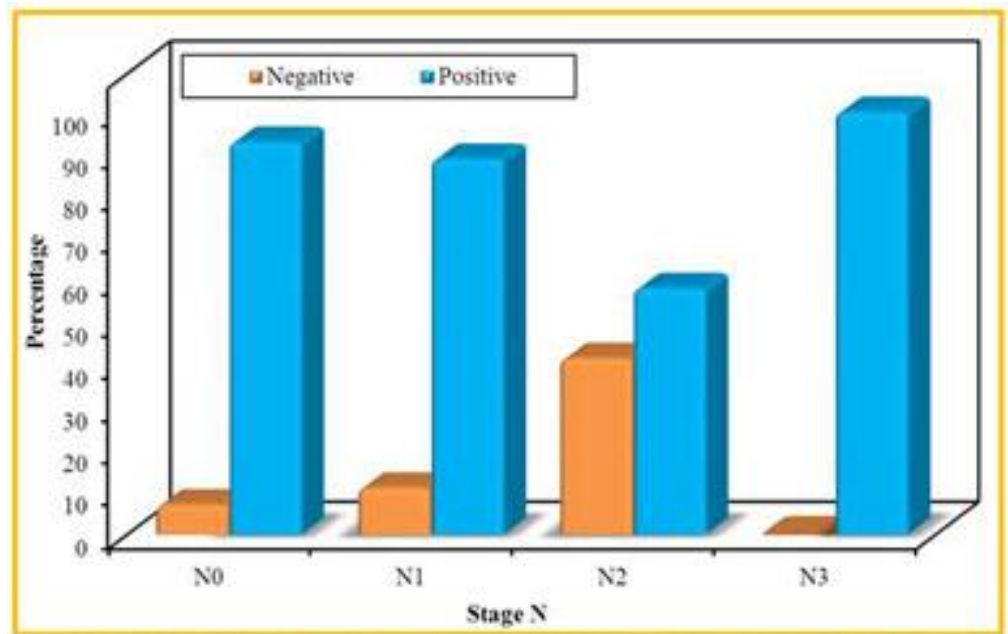

Figure (2): Relation between Cox2 expression and stage N.

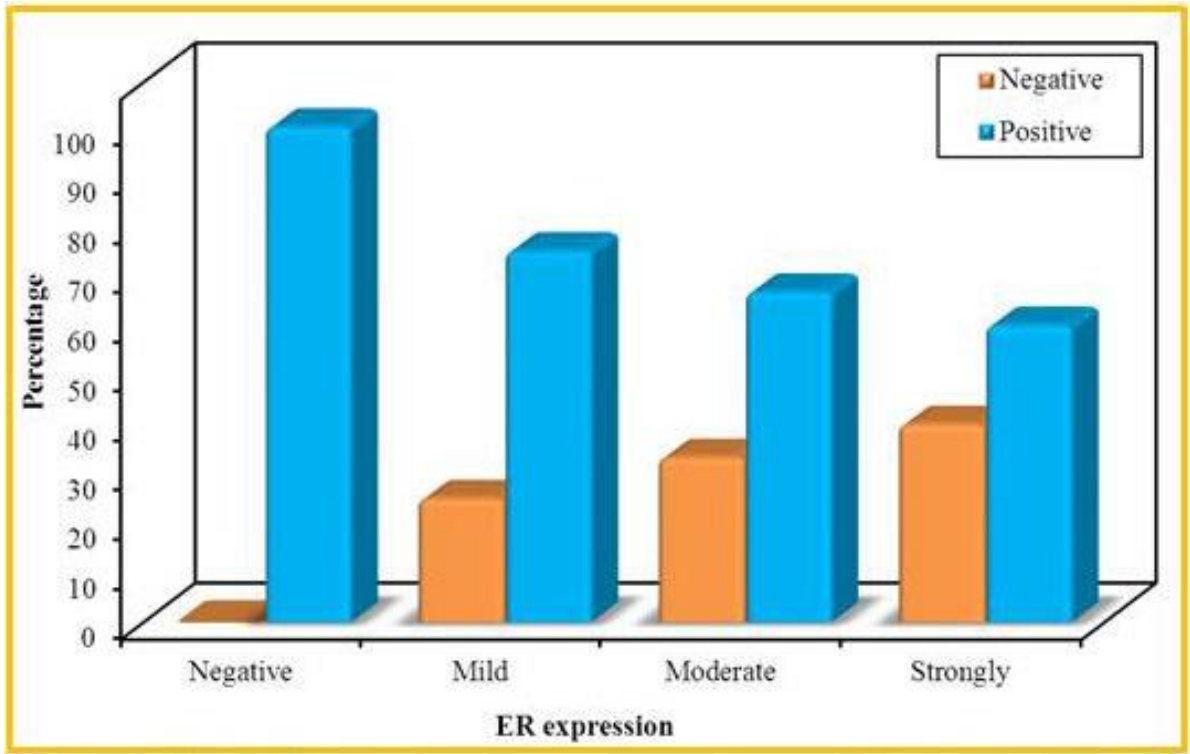

Figure (3): Relation between Cox 2 expression and ER expression.

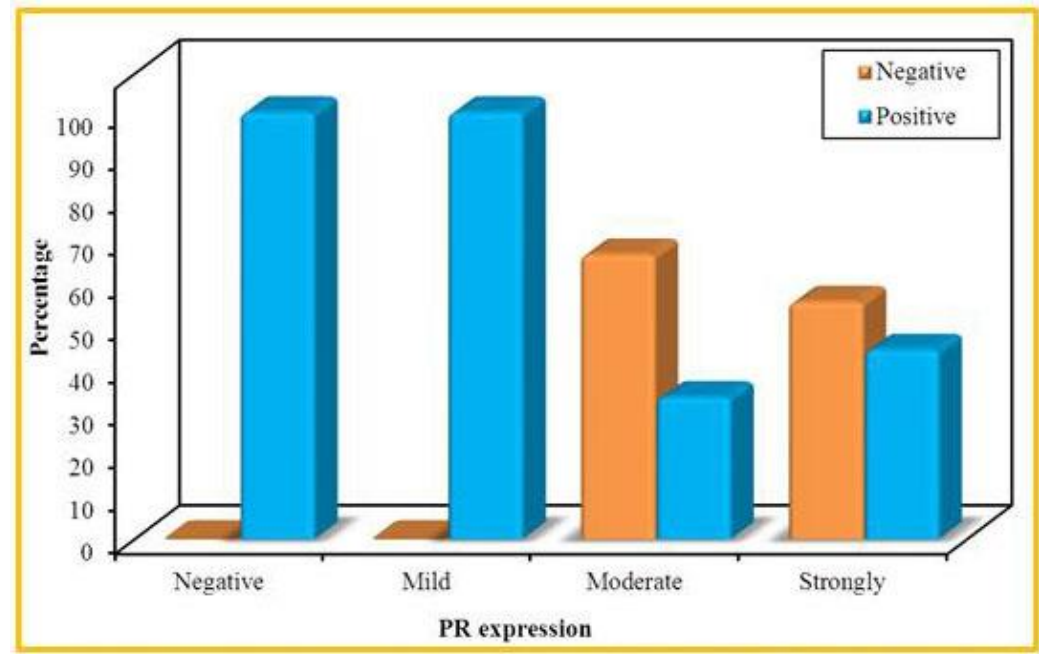

Figure (4): Relation between Cox 2 expression and PR expression. 


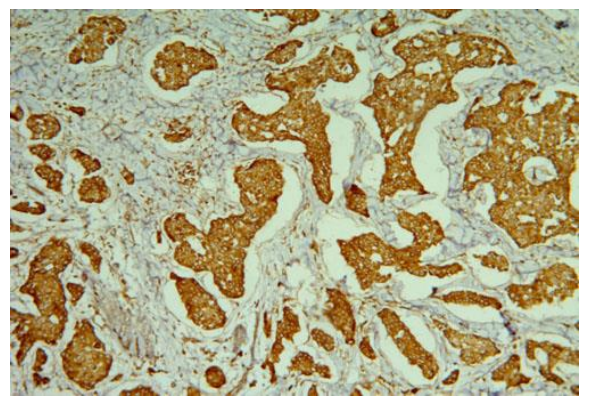

Figure (5): invasive duct carcinoma, showed strong cytoplasmic COX2expression in tumor cells (IHC X 100).

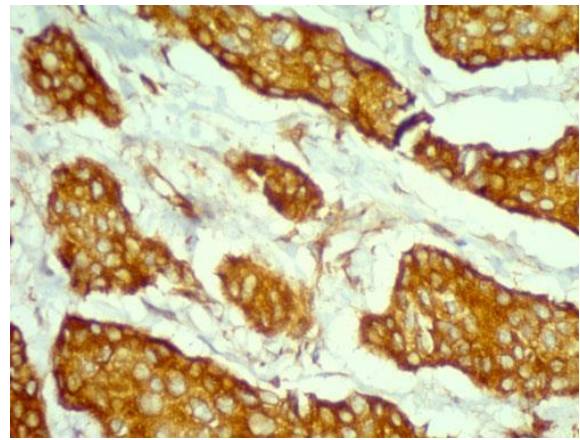

Figure (6): High power of previous case shows strong cytoplasmic expression of cox 2 in tumor cell (IHC X400).

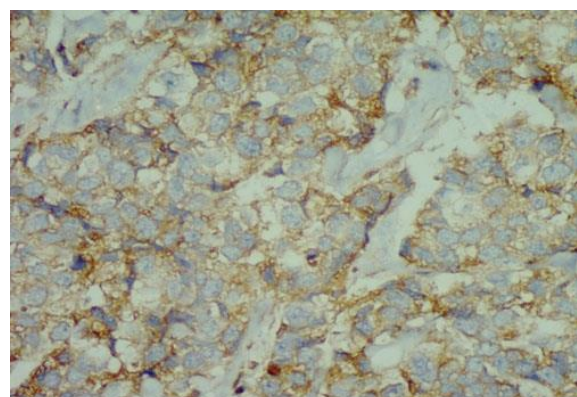

Figure (7): IDC, shows mild cytoplasmic expression of COX2 in tumor cells (IHC X400).

\section{DISCUSSION}

Breast cancer is the second leading cause of cancer-related death among women after lung cancer all over the world ${ }^{(\mathbf{1 7})}$. Breast cancer represents $38.8 \%$ of cancers occurring in Egyptian females in 2014 and it increases linearly as population increase ${ }^{(\mathbf{1 8 )}}$.

Evidence indicates a role for inflammation in the development of different steps of cancer progression; the incidence of several cancers is tightly associated to inflammation such as colon, breast, and prostate cancers ${ }^{(\mathbf{1 9})}$.

The aim of the current study was to evaluate IHC expression of COX-2 in tumor cells and their effect on prognosis of breast cancer.

In this study, cases were females of age ranging from 25 to 77 years. The mean age of the cases studied was 52 years, while the median age was 54 years. These results are comparable to those reported by Jana et al. ${ }^{(20)}$ who made studied Cox 2 in $123 \mathrm{fe}-$ male of primary breast carcinoma. They reported that the mean age was $50.78 \pm 8.81$ years with range of $37-$ 75 years and the median age was 50 years.

For histologic type, the current study denotes that most of studied cases were IDC (NST) (70.0\%). This agrees with Rakha et al. ${ }^{(21)}$ who reported that the majority ( $60 \%$ to $75 \%$ ) of breast cancers have no special type of characteristics.

In this study, about two thirds of the cases showed in situ component (DCIS\LCIS) (66\%). DCIS, the preinvasive form of breast cancer, forms a major part of breast surgery practice because of its liability to detection via screening mammography Half et al. ${ }^{(\mathbf{1 2})}$.

For tumor grade, grade II (moderately differentiated) tumors were found to represent the highest percentage $(74.0 \%)$ of cases in the current research. Tumor grade is an important determinant of breast cancer outcome and complementary to lymph node (LN) stage through the ability to influence the outcome of patients in different LN stage categories. Studies have also demonstrated that grade is an independent prognostic factor in specific subgroups of breast cancer patients ${ }^{(22)}$.

The tumor size in this study ranged from 1 to $8 \mathrm{~cm}$ with a mean of $3.73 \mathrm{~cm}$ and a median of $3.5 \mathrm{~cm}$. For node-negative patients, tumor size is the most powerful prognostic factor and is routinely used to make adjuvant treatment decisions ${ }^{(23)}$.

About one third of the studied cases showed LVI invasion (32.0\%). The existence of LVI may help identify who is at increased risk for axillary lymph 
node and distant metastasis ${ }^{(24)}$. Although LVI has been accepted as an important prognostic factor in patients with lymph node-negative invasive breast cancer (25), LVI is controversial regarding prognostic value in patients with lymph node-positive breast cancer ${ }^{(26)}$.

In the current study, Cox 2 expression using immuno-histochemical staining was detected in the majority of the studied cases $(86 \%)$ regardless their histological type with /without in situ component. Some studies reported its over expression ranged from $63-95 \%{ }^{(27)}$. On the other hand, other studies showed lower percentage that ranged from $37-56 \%{ }^{(17)}$ and these differences between various studies may be due to different cutoffs and variations or differences in scoring systems ${ }^{(28) .}$

On correlation of COX-2 expression with clinicopathological variables, no significant statistical association between COX-2 expression in tumor cells and other clinicopathologic parameters including age, tumor size, tumor location, histologic type, tumor grade and lymphovascular invasion. These results agree with Kelly et al. ${ }^{(29)}$ who studied the expression of COX-2 by immunohistochemistry in 106 primary breast carcinoma specimens and found that no statistically significant correlation between cox 2 and tumor size, grade, histology and nodal status. Also, Ranger et al. ${ }^{(30)}$ observed a significant association between COX-2 overexpression and distant metastasis, but no significant association with any other clinical or pathological variable.

In contrast, some of these studies showed that COX-2 expression was associated with larger tumor size, high nuclear grading, poor differentiation, and lymphovascular invasion ${ }^{(28)}$. Shim et al. ${ }^{(7)}$ observed COX-2 over expression in 46 out of 64 (72\%) breast cancers and studied the correlation of COX-2 expression with various clinic pathologic parameters, including tumor size, lymph node status, stage, ER and PR expression, and nuclear and histologic grade. Larger size and advanced stage in tumors were significantly correlated with COX-2 over expression.

In this study no significant statistical correlation could be detected between COX2 expression and insitu component. This result agrees with Kelly et al. ${ }^{(29)}$ and Ranger et al. ${ }^{(30)}$. Also, the study by Jana et al. ${ }^{(20)}$ found that the histology of lobular carcinoma and ductal carcinoma in situ did not show any significant difference in COX-2 expression.

However, in the present work majority of cases reported in situ component showed COX2 expression $(87.9 \%)$. Two third of the cases with in situ component expressed COX 2 with score 4 . The study by Half et al. ${ }^{(12)}$ hypothesized that COX-2 up-regulation is an early event in carcinogenesis and thus is overexpressed in pre-invasive lesions, Women with initial DCIS lesions that express COX-2 have a higher risk for developing subsequent invasive cancer in compari- son to those with COX-2 negative DCIS ${ }^{(31)}$. The discrepancy between different results may be due to different methods used for evaluation of COX-2 expression and COX-2 scoring as well as small number of studied cases.

The present work revealed that high significant correlation between lymph node positive cases and $\mathrm{COX}-2$ expression $(\mathrm{P}=0.009)$. Also, high significant association was detected between COX-2 expression and pathologic stage of lymph node $(\mathrm{P}=0.01)$.

Several studies show significant correlation between COX2 expression and lymph node metastasis. For example, Costa et al. ${ }^{(32)}$ indicated that significant association could be found between COX2 expression and lymph node metastasis, which may suggest that COX-2 over expression is associated with aggressive features and poor prognostic parameters in breast cancer.

According to the current work, there is high significant association between COX2 over expression and $\mathrm{ER}$ negative cases $(\mathrm{P}=0.001)$ and $\mathrm{PR}$ negative cases $(\mathrm{P}<0.001)$. These results agree with Kargi et al. ${ }^{(33)}$ and Jana et al. ${ }^{(20) .}$

The study of Jana et al. ${ }^{(20)}$ found that high levels of COX-2 expression correlated with absence of ER and PR expression. COX-2 expression in ER negative cell lines is also associated with mutated RAS. Increased expression of this protein has been associated with reduced estrogen dependence in breast cells. Both PKC and mutated RAS have been associated with an increased metastatic potential in cell lines ${ }^{(20) .}$

It is established that PR negative, ER negative cases of breast cancer are known to be of bad prognosis and that agree with the bad prognostic behavior of COX-2 in breast cancer ${ }^{(20)}$.

\section{CONCLUSION}

In this study expression of COX2 is not statistically significant with clinico-pathological parameters; the age, histologic type, tumor size or grade. However it is highly significant with cases of negative ER \&PR as well as lymph nodal metastasis. This indicates it is associated with poor prognosis. This observation may paints to the need to consider anti COX2 treatment modalities in treating the patient.

\section{REFERENCES}

1. Chen XS, Ma CD, Wu JY et al. (2010): Molecular subtype approximated by quantitative estrogen receptor, progesterone receptor and Her2 can predict the prognosis of breast cancer. Tumor., 96(1): 103-10.

2. Chowa LW, Yip AY, Loo WT et al. (2008): Celecoxib anti-aromatase neoadjuvant (CAAN) trial for locally advanced breast cancer. J Steroid Biochem Mol Biol., 111:13-17.

3. Jana D, Sarkar DK, Maji A et al. (2012): Can cyclooxygenase-2 be a useful prognostic and risk stratification 
marker in breast cancer. Journal of the Indian Medical Association, 110(7): 429-433.

4. Greenhough A, Smartt HJ, Moore AE et al. (2009): The COX-2/PGE 2 pathway: key roles in the hallmarks of cancer and adaptation to the tumour microenvironment. Carcinogenesis, 30(3): 377-386.

5. Misra $\mathbf{S}$ and Sharma K (2014): COX-2 signaling and cancer new players in old arena. Curr Drug Targets., 15:347-359.

6. Khan Z, Khan N, Tiwari RP et al. (2011): Biology of Cox-2: an application in cancer therapeutics. Curr Drug Targets, 12:1082-1093.

7. Shim JY, An HJ, Lee YH et al. (2003): Overexpression of cyclooxygenase -2 is associated with breast carcinoma and its poor prognostic factors. Mod pathol., 16:11991204.

8. Yiannakopoulou EC (2015): Aspirin and NSAIDs for breast cancer chemoprevention. European Journal of Cancer Prevention, 24(5): 416-421.

9. Lakhani SR, Ellis IO, Schnitt SJ et al. (2012): WHO Classification of Tumours of the Breast. Fourth ed. IARC; Lyon.

10. Elston CW and Ellis IO (1991): Pathological prognostic factors in breast cancer. I. The value of histological grade in breast cancer: experience from a large study with long -term follow-up. Histopathology, 19(5), 403410.

11. Hammond ME, Hayes DF, Dowsett M et al. (2010): American Society of Clinical Oncology/College of American Pathologists Guideline Recommendations for Immunohistochemical Testing of Estrogen and Progesterone Receptors in Breast Cancer Arch Pathol Lab Med., 134 (7): 48-72.

12. Half E, Tang XM, Gwyn K et al. (2002): Cyclooxygenase-2 expression in human breast cancers and adjacent ductal carcinoma in situ. Cancer research, 62(6): 1676-1681.

13. Kostopoulos I, Arapantoni-Dadioti P, Gogas $H$ et al. (2006): Evaluation of the prognostic value of HER-2 and VEGF in breast cancer patients participating in a randomized study with dose-dense sequential adjuvant chemotherapy. Breast cancer research and treatment, 96(3): 251-261.

14. Kirkpatrick LA and Feeney BC (2013): A simple guide to IBM SPSS statistics for version 20.0. Student ed. Belmont, Calif.: Wadsworth, Cengage Learning.

15. Kotz S, Balakrishnan N, Read CB et al. (2006): Encyclopedia of statistical sciences. 2nd ed. Hoboken, N.J.: Wiley-Interscience.

16. Dawson B and Trapp R (2001): Basic and Clinical Biostatistics. In: large medical books. Third edition. Oxford, London, Boston. 87.

17. Jemal A, Bray F, Center MM et al. (2011): Global cancer statistics. CA Cancer J Clin., 61(2): 69-90.

18. Ibrahim AS, Khaled HM, Mikhail NN et al. (2014): Cancer incidence in Egypt: results of the national population-based cancer registry program. Journal of cancer epidemiology, 2014.
19. Coussens LM and Werb $Z$ (2002): Inflammation and cancer. Nature, 420(6917): 860-867.

20. Jana D, Sarkar DK, Ganguly S et al. (2014): Role of Cyclooxygenase 2 (COX-2) in Prognosis of Breast Cancer. Indian J Surg Oncol., 5(1): 59-65.

21. Rakha EA, Reis-Filho JS, Baehner F et al. (2010): Breast cancer prognostic classification in the molecular era: the role of histological grade. Breast Cancer Res., 12 (4): 207

22. Rakha EA, El-Sayed ME, Lee AH et al. (2008): Prognostic significance of nottingham histologic grade in invasive breast carcinoma. J Clin Oncol., 26 (19): 31533158.

23. Cianfrocca M and Goldstein LJ (2004): Prognostic and predictive factors in early-stage breast cancer. Oncologist, 9(6): 606-616.

24. Woo CS, Silberman H, Nakamura SK et al. (2002): Lymph node status combined with lymphovascular invasion creates a more powerful tool for predicting outcome in patients with invasive breast cancer. Am J Surg., 184:337-340.

25. Yildirim $\mathbf{E}$ and Berberoglu $\mathbf{U}$ (2007): Lymph node ratio is more valuable than level III involvement for prediction of outcome in node-positive breast carcinoma patients. World J Surg., 31:276-289.

26. MacGrogan G, Desrousseaux M, de Mascarel I (2006): Prognostic value of Mib1 in a tissue microarray of 855 invasive breast carcinomas; 5th European Breast Cancer Conference. https://www.uicc.org/5th-europeanbreast-cancer-conference

27. Zhang XH, Huang DP, Guo GL et al. (2008): Coexpression of VEGF-C and COX-2 and its association with lymphangiogenesis in human breast cancer. BMC Cancer, 8: 4.

28. Singh-Ranger G, Salhab M, Mokbel K (2008): The role of cyclooxygenase- 2 in breast cancer: review. Breast Cancer Res Treat., 109(2): 189-198.

29. Kelly LM, Hill AD, Kennedy $\mathrm{S}$ et al. (2003): Lack of prognostic effect of Cox-2 expression in primary breast cancer on short-term follow-up. Eur J Surg Oncol., 29(9): 707-710.

30. Ranger GS, Thomas V, Jewell A et al. (2004): Elevated cyclooxygenase-2 expression correlates with distant metastases in breast cancer. Anticancer Res., 24(4): 2349-2351.

31. Kerlikowske K, Molinaro AM, Gauthier ML et al. (2010): Biomarker expression and risk of subsequent tumors after initial ductal carcinoma in situ diagnosis. J Natl Cancer Inst., 102(9):627-37.

32. Costa C, Soares R, Reis-Filho JS et al. (2002): Cyclooxygenase 2 expression is associated with angiogenesis and lymph node metastasis in human breast cancer. Journal of clinical pathology, 55(6): 429-434.

33. Kargi A, Uysal M, Bozcuk $H$ et al. (2013): The importance of COX-2 expression as prognostic factor in early breast cancer. J BUON., 18(3): 579-84. 